\title{
Nanofabrication of doped, complex oxides
}

\author{
Gordon H. Waller ${ }^{\mathrm{a})}$ \\ Department of Materials Science and Engineering, Virginia Polytechnic Institute and State University, \\ Blacksburg, Virginia 24061
}

\author{
Aaron Stein \\ Center for Functional Nanomaterials, Brookhaven National Laboratory, Upton, New York 11973 \\ Jeremiah T. Abiade \\ Department of Mechanical and Industrial Engineering, University of Illinois at Chicago, Chicago, \\ Illinois 60607
}

(Received 10 August 2011; accepted 20 November 2011; published 15 December 2011)

\begin{abstract}
Complex oxides have many promising attributes, including wide band gaps for high temperature semiconductors, ion conducting electrolytes in fuel cells, ferroelectricity and ferromagnetism. Bulk and thin film oxides can be readily manufactured and tested however these physically hard and chemically inert materials cannot be nanofabricated by direct application of conventional methods. In order to study these materials at the nanoscale there must first be a simple and effective means to achieve the desired structures. Here we discuss the use of pulsed laser deposition at room temperature onto electron beam lithography defined templates of poly methyl methacrylate photoresist. Following a resist liftoff in organic solvents, a heat treatment was used to crystallize the nanostructures. The morphology of these structures was studied using scanning electron microscopy and atomic force microscopy. Crystallinity and composition as determined by $\mathrm{x}$ ray diffraction and photo-electron spectroscopy respectively is reported for thin film analogues of the nanostructured oxide. The oxide studied in this report is $\mathrm{Nb}$ doped $\mathrm{SrTiO}_{3}$, which has been investigated for use as a high temperature thermoelectric material; however the approach used is not materials-dependent. (C) 2012 American Vacuum Society. [DOI: 10.1116/1.3669645]
\end{abstract}

\section{INTRODUCTION}

Many oxide materials have interesting properties in bulk form; however their complex stoichiometries, high melting temperatures, and resistance to wet chemical etching limit the capability to easily fabricate and study their nanostructure counterparts. One oxide of particular interest is $\mathrm{Nb}$ doped $\mathrm{SrTiO}_{3}$ (Nb-STO), which has demonstrated potential as a high performance thermoelectric material in both bulk and thin film forms. ${ }^{1-3}$ While changes in stoichiometry can manipulate the governing terms that define the figure of merit, Z [Eq. (1)] below,

$$
Z=\frac{\sigma S^{2}}{k},
$$

where $\sigma$ is electrical conductivity, $S$ is the Seebeck coefficient and $k$ is thermal conductivity these terms are typically interrelated, preventing isolated maximization or minimization to increase Z. ${ }^{4}$ Nanostructuring of thermoelectric materials, specifically as one dimensional nanostructures (nanorods and nanowires), has already been demonstrated in some materials systems to achieve a reduction in the thermal conductivity $k$ without decreasing $\sigma$ or $S$ by restricting phonon migration. ${ }^{5,6}$ Enhancement of thermoelectric performance in bulk Nb-STO with nanoscale inclusions has also been attributed to a reduction in phonon migration. ${ }^{7-9}$ In this work, we demonstrate the capability to fabricate nanostructured $\mathrm{Nb}-\mathrm{STO}$ of various

\footnotetext{
a) Author to whom correspondence should be addressed; electronic mail: gwaller@vt.edu
}

geometries. The physical properties of the structures will be investigated in future studies.

One dimensional oxide nanostructures can be readily fabricated by wet chemical synthesis, but the final length and diameter is dependent on the extent of Ostwald ripening. ${ }^{10,11}$ Oxides formed in this way are also typically undoped. Top down approaches have also been investigated in which a thin film of the desired stoichiometry is shaped via ion milling. However, this approach can cause undesirable crystallographic damage, ion implantation which manipulates the electrical properties, and re-deposition of the milled material. ${ }^{12-14}$ While strategies to mitigate these drawbacks exist, they require additional process complexity. ${ }^{15,16}$ Template assisted growth in which a negative of the desired nanostructure is used to modulate the deposition of the oxide onto a substrate has produced an incredible variety of techniques as both the template and deposition methodology have been developed elsewhere in materials science research. Ideally, templates should be easily placed and patterned before and easily removed after deposition, all while being robust enough to ensure accurate reproduction of the negative template features. Lithography easily meets this criterion but for some deposition techniques requires several processing steps such as deep reactive ion etching to transfer the template structure from the photoresist to the substrate, which is then itself used as the template. The Dravid group has been especially prolific in combining this type of template with oxide sols. ${ }^{17-19}$

A nanostructuring technique is needed that allows control of stoichiometry, material dimension and spatial arrangement. 
Here we investigate the use of electron beam lithography (EBL) to pattern resists on oxide substrates. After development, the resist was used directly as a deposition mask during room temperature pulsed laser deposition (PLD). Subsequent liftoff of the resist in a solvent bath and post deposition heat treatment results in a high yield, simple and effective process for producing crystalline nanostructures.

\section{EXPERIMENT}

Figure 1 is a graphical depiction of the experimental procedure, and will be referred to in this section. Templates were written into 4 wt.\% poly methyl methacrylate resists dissolved in anisole (PMMA-A4) using EBL (JEOL JBX6300FS). The PMMA-A4 resist was coated onto single crystalline (100) oriented $\mathrm{SrTiO}_{3}$ (STO) substrates (CrysTec $\mathrm{Gmbh}$ ). The oxide substrates were first cleaned in acetone, isopropanol and methanol and then subjected to an oxygen plasma asher for 30 seconds. The PMMA-A4 was then spun on at $3000 \mathrm{rpm}$ for a final thickness of about $175 \mathrm{~nm}$, and then baked for 5 mins at $180{ }^{\circ} \mathrm{C}$, the resist coated substrate is shown in Fig. 1(a). Following the resist bake, a conductive polymer "ESPACER" was spun on and baked using the same speed and temperature. The ESPACER top layer prevented charge buildup on the surface of the insulating substrates during exposure in the EBL tool. The patterns used were either trenches with horizontal widths ranging from 10 $\mathrm{nm}$ to $1 \mu \mathrm{m}$ and lengths of $500 \mu \mathrm{m}$, or square pits with side widths of $50 \mathrm{~nm}$ and $100 \mathrm{~nm}$. Following patterning, the resist was developed in a solution of methyl isobutyl ketone in isopropanol (MIBK) for one minute, and then rinsed in isopropanol, leaving bare areas of substrate where electron irradiated resist made soluble in the developer solution. An exposed and developed substrate with a trench style pattern is shown in Fig. 1(b). The electron beam was set at a constant $4 \mathrm{nA}$ current and $100 \mathrm{keV}$ accelerating voltage. Dose was manipulated along with pattern dimension and feature density to achieve the final templates.

Template filling occurred under high vacuum $\left(10^{-6}\right.$ Torr $)$ at room temperature using PLD. A $248 \mathrm{~nm}$ wavelength $\mathrm{KrF}$ Excimer laser (GAM Laser EX 350) was used to ablate a $\mathrm{SrTi}_{.8} \mathrm{Nb}_{.2} \mathrm{O}_{3}(5 \mathrm{~atm} \% \mathrm{Nb})$ sintered target. A template with deposited film is shown in Fig. 1(c). Laser parameters were kept constant at $2 \mathrm{~J} / \mathrm{cm}^{2}$ with a repetition rate of $5 \mathrm{~Hz}$. After determining the growth rate using a partially masked substrate and a profilometer, the number of laser pulses was used to adjust the thickness of the deposited material and height of the nanostructures. Following PLD the substrates were placed in an acetone bath at $90{ }^{\circ} \mathrm{C}$ for ten minutes and then sonicated for ten seconds. Figure 1(d) shows the freestanding oxide nanostructures after the template is dissolved. Following the liftoff procedure, the substrates and nanostructures were annealed under high vacuum at $650{ }^{\circ} \mathrm{C}$ for one hour and then cooled at $5{ }^{\circ} \mathrm{C} / \mathrm{min}$ to room temperature. The nanostructures were coated with $2-3 \mathrm{~nm}$ of $\mathrm{Pt} / \mathrm{Au}$ for scanning electron microscopy (SEM) (FEI Quanta 600 FEG) imaging to observe general morphology. Additional uncoated nanostructures were studied using atomic force microscopy (AFM) in contact mode (VEECO Multimode AFM) for height measurements.

Thin film analogues were deposited at room temperature using identical laser parameters as the template filling experiments to study crystallization and film composition. The room temperature deposited thin films were subjected to the same annealing heat treatments as their nanostructured counterparts. Films deposited at $650{ }^{\circ} \mathrm{C}$ were also studied to serve as a reference for the annealed films. X-ray photoelectron spectroscopy (XPS) (PHI Quantera SXM) sputter depth composition profiling, X-ray diffraction (XRD) (Phillips Xpert Pro with $\mathrm{Cu}-\mathrm{K}_{\alpha} \mathrm{x}$ ray source) and sheet resistance measurements using the Van der Pauw method (Keithley 4200 SCS) were used to characterize the thin film samples.

\section{RESULTS AND DISCUSSION}

\section{A. Oxide structures}

Templates fabricated using EBL on oxide substrates produced a substantial increase in final dimensions compared to the expected feature width. For a template with a nominal dimension of $10 \mathrm{~nm}$ (as defined in the pattern generation software) a final dimension of $110 \mathrm{~nm}$ was achieved. These dimensions were accurately conveyed to the deposited oxide. Figure 2 is an SEM image showing the dimensions of the
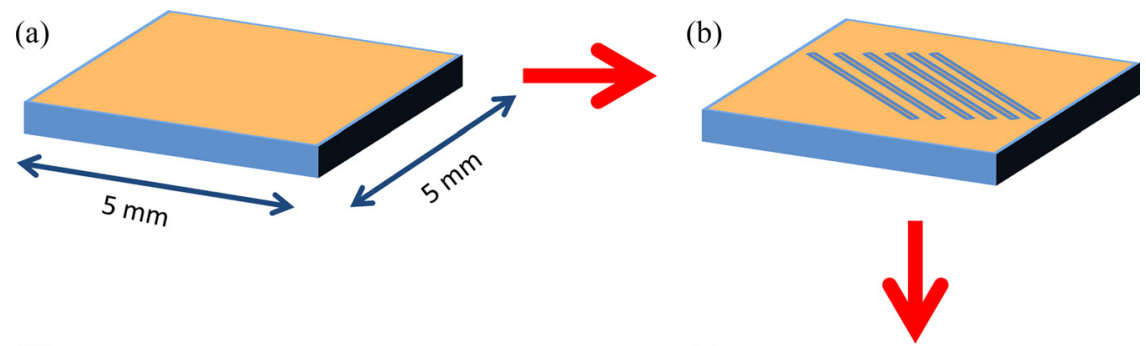

(d)

(c)
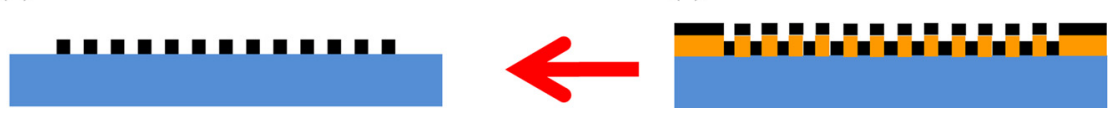

FIG. 1. (Color online) Experimental procedure used in the template guided deposition of Nb-STO at various steps-(a) shows the PMMA coated substrate before EBL, (b) shows the trenches in the PMMA after EBL exposure and developing in MIBK-IPA, (c) C shows the template filled with NbSTO after pulsed laser deposition (PLD) and (d) D shows the freestanding structures after liftoff of the resist in the solvent bath. 


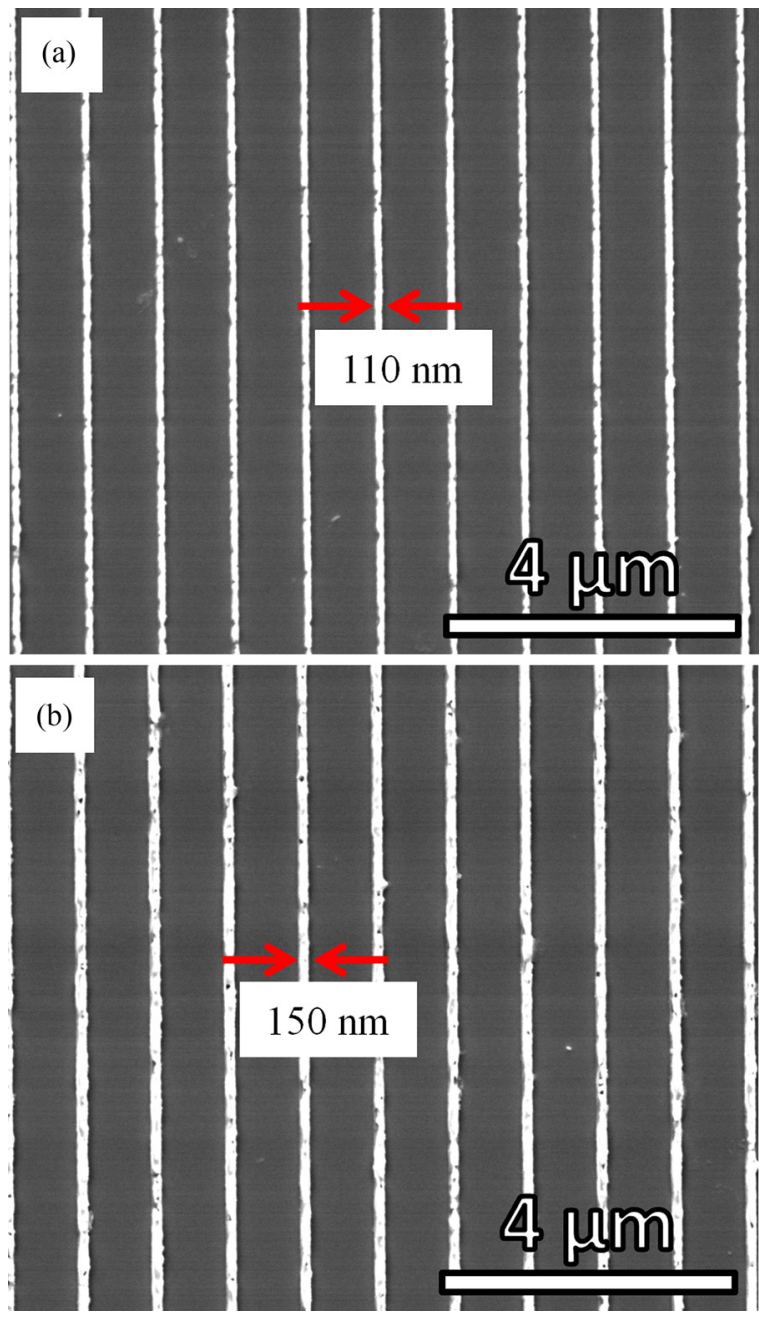

FIG. 2. (Color online) Scanning electron microscopy (SEM) image of $\mathrm{Nb}$ STO wires produced using a $10 \mathrm{~nm}$ (a) and $30 \mathrm{~nm}$ (b) nominal width and $1 \mu \mathrm{m}$ pitch. Images were taken at $5 \mathrm{kV}$ with an $8 \mathrm{~mm}$ working distance after $\mathrm{Au} / \mathrm{Pt}$ coating of samples, and SEM measured dimensions are indicated by the arrows.

oxide nanostructures using $10 \mathrm{~nm}$ and $30 \mathrm{~nm}$ nominal wire diameters. A dose of $5200 \mu \mathrm{C} / \mathrm{cm}^{2}$ was required to produce the features shown in Figs. 2-4, which is significantly larger than the typical dose of $1000-2000 \mu \mathrm{C} / \mathrm{cm}^{2}$ used for PMMA-A4 on silicon substrates. Using a box pattern at the same dose as for the PLD filled trenches shown in Fig. 2 produced nanodots after deposition and liftoff. Figure 3 shows filled box style templates with nominal dimensions of $50 \mathrm{~nm}$ and $100 \mathrm{~nm}$. The actual dimension for each structure is indicated by the arrows.

Lower doses produced heavily scummed trenches after developing. Both the expansion in template dimension and the high dose required for complete exposure can be explained by a phenomenon known as proximity dosing which is explained as follows. In a typical EBL experiment, electrons penetrate the sample normal to the substrate surface and assuming a high enough electrical conductivity follow a path to ground through the bulk of the sample, exposing the resist directly in the path of electron beam. The depth of the initial penetration and the subsequent
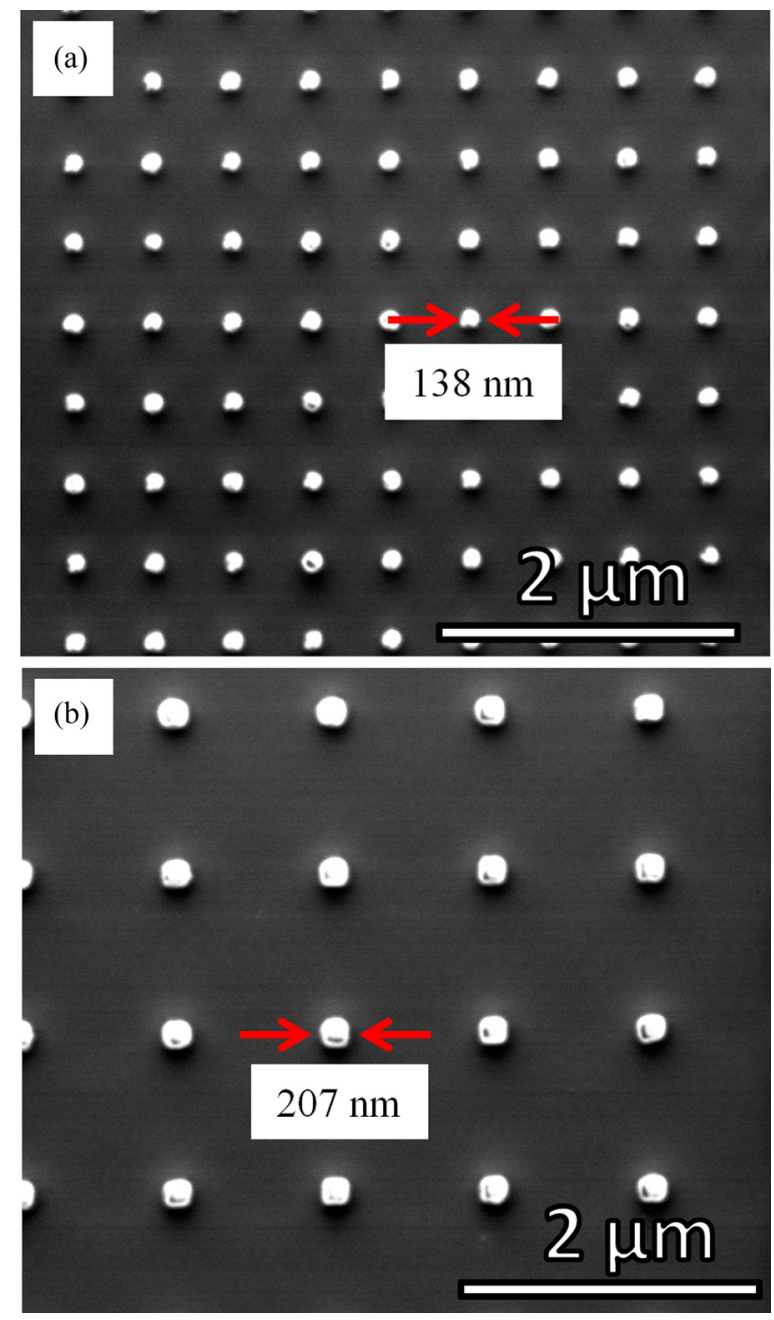

FIG. 3. (Color online) Scanning electron microscopy (SEM) image of NbSTO nanodots produced using a box pattern of $50 \mathrm{~nm}$ side width $500 \mathrm{~nm}$ pitch (a) and $100 \mathrm{~nm}$ side width $1 \mu \mathrm{m}$ pitch (b). Images were taken at $5 \mathrm{kV}$ with an $8 \mathrm{~mm}$ working distance after $\mathrm{Au} / \mathrm{Pt}$ coating of samples, and SEM measured dimensions are indicated by the arrows.

electron-sample interactions (such as the release of secondary electrons or the backscattering of the incident beam) are highly dependent on the accelerating voltage of the electron beam as well as the atomic weight of the substrate. Furthermore, an insulating substrate will develop a localized surface around the electron irradiated area leading to additional problems with beam deflection. This is avoided with the use of the conductive polymer top layer ESPACER which disperses the localized charge. For a doped semiconductor such as silicon, the accelerating voltage, resist thickness and pattern densities have been optimized to avoid proximity dosing and produce features on the order of $10 \mathrm{~nm}$ using EBL. The substrates used in this report are nontraditional from an EBL point of view, as they are insulating and have a higher average atomic weight. The result is that the dose typically required to expose a resist on $\mathrm{Si}$ is inaccurate for our substrates. On STO, a portion of the electron beam is dispersed most likely by backscattering or localized charge concentration, exposing the resist in the near proximity to the designated area of irradiation. As a consequence, the high dose 

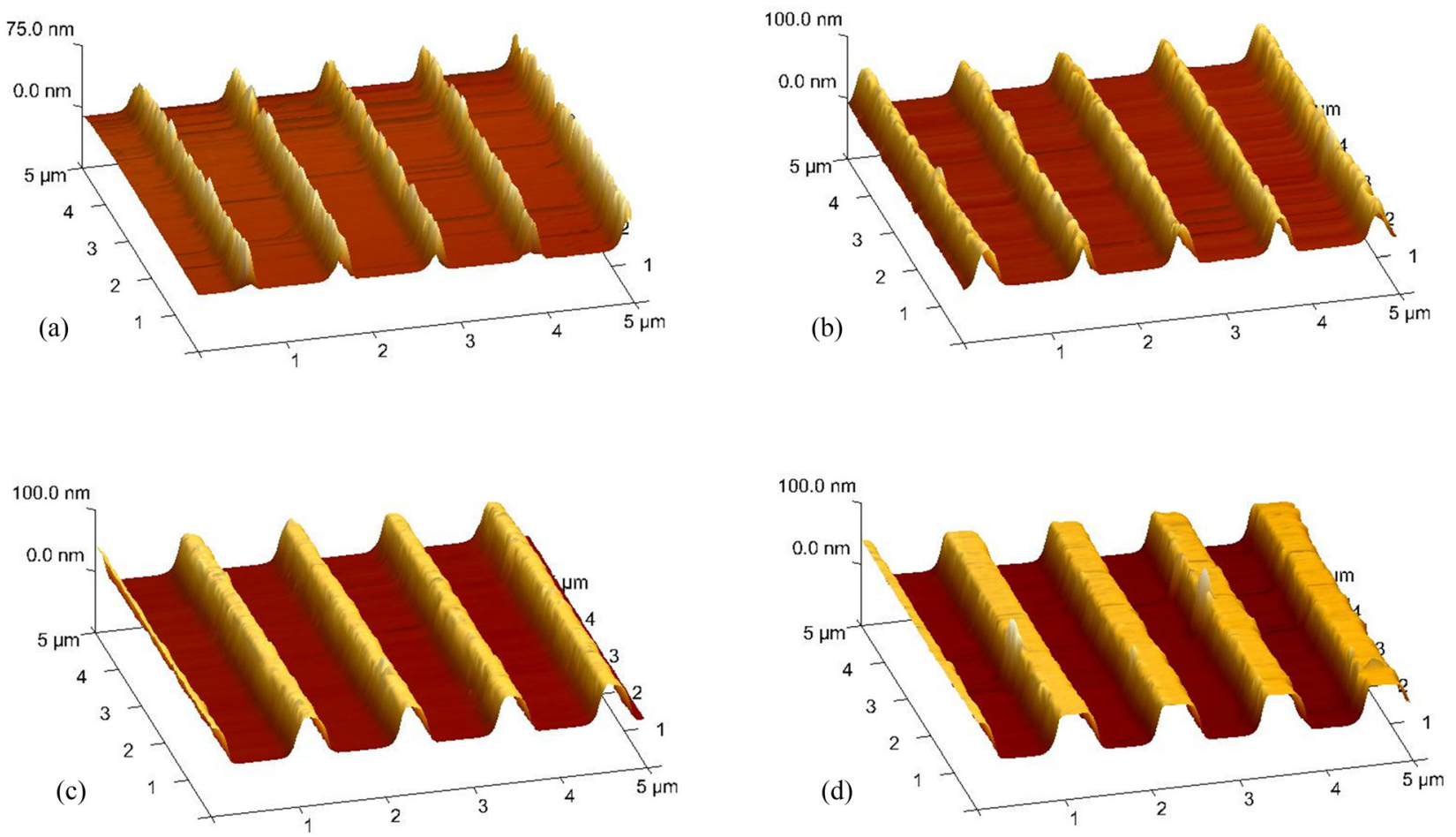

Fig. 4. (Color online) Contact mode atomic force microscopy (AFM) images of wire type nanostructures with actual widths of $100 \mathrm{~nm}$ (a), $190 \mathrm{~nm}$ (b), $290 \mathrm{~nm}$ (c) and $530 \mathrm{~nm}$ (d) as determined by scanning electron microscopy (SEM).

required to completely expose the resist causes an undesirable pattern broadening, which we minimized by using a large pitch of $1 \mu \mathrm{m}$ in the trench style templates. A careful design of the irradiation pattern or the use of lower accelerating voltages may alleviate this problem in future studies.

AFM imaging of the horizontal wire structures shows an increase in cross-sectional uniformity with increasing lateral dimension. Wires with lateral dimensions of $110 \mathrm{~nm}$ to 530 $\mathrm{nm}$ are shown in Fig. 4. The widths of each feature in Fig. 4 were determined by SEM and are given in the figure caption. All of the features shown in Fig. 4 have a height of approximately $60 \mathrm{~nm}$ after 2000 laser pulses, which is consistent with the PLD growth rate for a film. The length of the features is $500 \mu \mathrm{m}$. The jagged peaks observed for the $110 \mathrm{~nm}$ wires are partially due to the angle of the AFM tip; however the increasing smoothness on the tops of the widest wires indicates a more homogeneous filling of the template. This is possibly due to a reduced interaction of the deposited material with the template edge during deposition due to decreased aspect ratio of the pattern for wider features.

\section{B. Characterization of thin film analogues}

For the thin film analogues, $100 \mathrm{~nm}$ films of $\mathrm{Nb}-\mathrm{STO}$ were deposited on (100) oriented $\mathrm{LaAlO}_{3}$ (LAO) and STO (100) substrates. LAO substrates were used for XRD analysis to avoid an overlap between the Nb-STO film peaks and STO substrate peaks. STO substrates were used for the XPS analyzed films. The XRD results in Fig. 5 shows that the film deposited at room temperature has no crystalline peaks except for the LAO substrate; thus the film is possibly amorphous. After annealing the room temperature deposited film, a single diffraction peak emerges suggesting the film is highly textured. When compared to films deposited at high temperature $\left(650{ }^{\circ} \mathrm{C}\right)$ the annealed film diffraction peak is shifted towards LAO (200). This suggests a greater influence of the substrate lattice constant on the annealed film and supports the assumption that the annealed film is polycrystalline with some reorientation of its grains due to a compressive epitaxial strain. Using the Bragg relation as shown below [Eq. (2)]

$$
n \lambda=2 d \sin \theta
$$

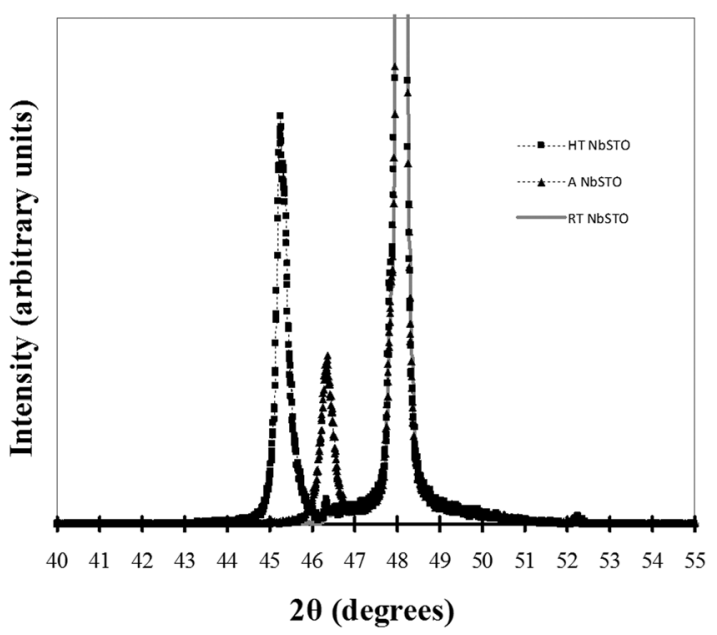

FIG. 5. X-ray diffraction (XRD) spectrum of Nb-STO films deposited at room temperature (RT NbSTO), room temperature followed by annealing (A NbSTO) and at high temperature (HT NbSTO) on $\mathrm{LaAlO}_{3}$ (100) substrates. 
TABLE I. Calculation of lattice spacing for room temperature deposited and unnealed film with only $\mathrm{LaAlO}_{3}$ (200) substrate peak (RT NbSTO), the crystalline peaks produced by annealing the room temperature deposited film (A NbSTO) and the high temperature deposited film (HT NbSTO).

\begin{tabular}{lcc}
\hline \hline Sample & $2 \theta$ (degrees) & $\mathrm{d}(\AA)$ \\
\hline RT NbSTO & - & - \\
A NbSTO & 46.35 & 3.92 \\
HT NbSTO & 45.25 & 4.01 \\
\hline \hline
\end{tabular}

where $\lambda$ is the wavelength of the diffracting $\mathrm{x}$ rays $(\sim 1.54 \AA$ for $\mathrm{Cu}-\mathrm{K}_{\alpha}$ ) and $\theta$ is the measured diffraction angle, the lattice spacing $d$ can be determined. For each diffraction peak observed in Fig. 5, the lattice spacing calculation is found in Table I. For the room temperature deposited, unannealed film (RT NbSTO) only the LAO (200) substrate peak is observed. While both films exhibit an increased lattice constant compared to the nominal lattice constant of undoped STO (3.905 $\AA$ ), which is expected from substitutional doping of the larger $\mathrm{Nb}$ atoms, the high temperature deposited film (HT NbSTO) is closest to the value reported elsewhere for PLD grown Nb-STO films at high temperature and vacuum conditions. $^{20,21}$

XPS sputter depth profiling of the annealed film shows a uniform distribution of $\mathrm{Nb}, \mathrm{Sr}, \mathrm{Ti}$ and $\mathrm{O}$ in atomic \% throughout the entire depth of the deposited oxide shown in Fig. 6. The films were first sputter cleaned to remove carbon and other impurities, and thus the first $5 \mathrm{~nm}$ of XPS data is not included. Sputter time was converted to sputter depth by assuming a constant sputter rate of the deposited film compared to a bare STO substrate. Sputter rate was determined by eroding a bare STO substrate with argon for 10 mins and the resultant depth of the sputter crater was measured with a stylus profilometer. The $\mathrm{Nb}$ signal dissipates at the substrate interface after sampling the $100 \mathrm{~nm}$ film, indicating that the post deposition heat treatment did not cause $\mathrm{Nb}$ diffusion into the STO substrate. Within the substrate both the Ti and $\mathrm{Sr}$ signals increase slightly suggesting that the $\mathrm{Nb}$ dopant may be replacing the A site cation ( $\mathrm{Sr}$ ) in addition to $\mathrm{B}$ site cation (Ti) in the perovskite structure. Sheet resistance measurements of the XRD analyzed films presented in Table II showed that the high temperature deposited films had electrical conductivities on the same order of magnitude as that

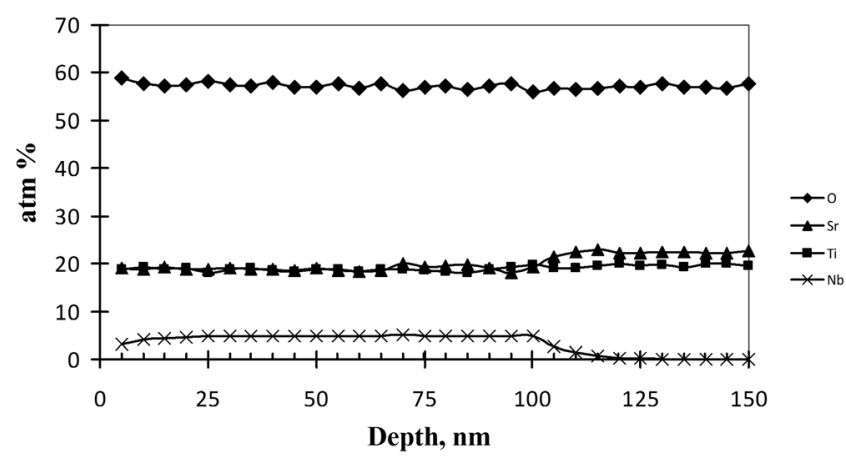

FIG. 6. X-ray photoelectron spectroscopy (XPS) sputter depth profile of an $\mathrm{Nb}-\mathrm{STO}$ film on $\mathrm{SrTiO}_{3}(100)$ substrate annealed at $650{ }^{\circ} \mathrm{C}$.
TABLE II. Sheet resistance values for high temperature deposited (HT NbSTO), room temperature deposited then annealed (A NbSTO) and room temperature deposited without annealing (RT NbSTO) films as measured by a Van der Pauw probe configuration.

\begin{tabular}{lcc}
\hline \hline Sample & Electrical Conductivity $(\Omega \mathrm{cm})$ & Standard Deviation \\
\hline RT NbSTO & $3.74 \mathrm{E}+02$ & $9.67 \mathrm{E}+01$ \\
A NbSTO & $1.43 \mathrm{E}+00$ & $6.08 \mathrm{E}-2$ \\
HT NbSTO & $1.81 \mathrm{E}-04$ & $2.65 \mathrm{E}-05$ \\
\hline \hline
\end{tabular}

reported in the literature for laser ablation deposited epitaxial Nb-STO films of the same nominal composition and similar experimental conditions. ${ }^{21}$ However, the annealed films have a higher sheet resistance, though lower than that of the unannealed film. The formation of oxygen vacancies in the high temperature deposited film (which was deposited under high vacuum) is a likely explanation for the lower sheet resistance. Likewise, if the larger resistivity of the annealed film is assumed to be due to a lower concentration of oxygen vacancies, then the total concentration of oxygen should be similar within the film and the substrate, which is supported by the XPS result shown in Fig. 6 .

\section{SUMMARY AND CONCLUSIONS}

The discussed templating technique has a resolution on the order of $100 \mathrm{~nm}$, and with appropriate heat treatment following deposition and liftoff can produce highly crystalline oxide nanostructures of the desired stoichiometry. In comparison to other templates filled via PLD, the minimum feature size is comparable if not superior, and the ease of the liftoff procedure makes the technique especially interesting for future studies in functional oxide heterostructures. ${ }^{22-24}$

\section{ACKNOWLEDGMENTS}

This work was partially supported by the United States National Science Foundation Grant No. NSF-BRIGE EEC0824340. A part of this research was conducted at the Center for Functional Nanomaterials, Brookhaven National Laboratory, which is supported by the U.S. Department of Energy, Office of Basic Energy Sciences, under Grant No. DEAC02-98CH10886.

${ }^{1}$ S. Ohta, T. Nomura, H. Ohta, and K. Koumoto, J. Appl. Phys. 97, 034106 (2005).

${ }^{2}$ K. H. Lee, S. W. Kim, H. Ohta and K. Koumoto, J. Appl. Phys. 100, 063717 (2006).

${ }^{3}$ Y. Wang, K. H. Lee, H. Ohta and K. Koumoto, J. Appl. Phys. 105, 103701 (2009).

${ }^{4}$ D. M. Rowe, Thermoelectrics Handbook: From Macro to Nano (CRC, Boca Raton, 2006) pp. 1.1-1.14.

${ }^{5}$ D. Li, Y. Wu, R. Fan, P. Yang, and A. Majumdar, Appl. Phys. Lett. 83, 15 (2003).

${ }^{6}$ A. Boukai, Y. Bunimovich, J. Tahir-Kheli, J. K. Yu, W. A. Goddard, and J. R. Heath, Nature 451, 06458 (2008).

${ }^{7}$ N. Wang, L. Han, H. He, Y. Ba, and K. Koumoto, J. Alloys Compd. 497 (2010).

${ }^{8}$ N. Wang, H. Li, Y. Ba, Y. Wang, C. Wan, K. Fujinami, and K. Koumoto, J. Electron. Mater. 39, 9 (2010).

${ }^{9}$ N. Wang, H. He, Y. Ba, C. Wan, and K. Koumoto, J. Ceram. Soc. Jpn. 118, 11 (2010). 
${ }^{10}$ J. J. Urban, W. S. Yuan, Q. Gu, and H. Park, J. Am. Chem. Soc. 124, 7 (2002).

${ }^{11}$ U. A. Joshi and J. S. Lee, Small 1, 12 (2005).

${ }^{12}$ V. Komanicky, H. Iddir, K. C. Chang, A. Menzel, G. Karapetrov, D. C. Hennessy, P. Zapol, and H. You, Electrochim. Acta 55 (2010).

${ }^{13}$ A. Stanishevsky, B. Nagaraj, J. Melngailis, R. Ramesh, L. Khriachtchev, and E. McDaniel, J. Appl. Phys. 92, 6 (2002).

${ }^{14}$ A. Schilling, T. Adams, R. M. Bowman, and J. M. Gregg, Nanotechnology 18, 035301 (2007).

${ }^{15}$ V. Nagarajan, A. Stanishevsky, and R. Ramesh, Nanotechnology 17 (2006).

${ }^{16}$ M. Hambe, S. Wicks, J. M. Gregg, and V. Nagarajan, Nanotechnology 19, 175302 (2008).
${ }^{17}$ Z. Pan, S. K. Donthu, N. Wu, S. Li, and V. P. Dravid, Small 2, 2 (2006).

${ }^{18}$ V. P. Dravid, J. Mater. Chem 19, 4295 (2009).

${ }^{19}$ T. Sun, Z. Pan, V. P. Dravid, Z. Wang, M. F. Yu, and J. Wang, Appl. Phys. Lett. 89, 163117 (2006).

${ }^{20} \mathrm{~K}$. Fukushima and S. Shibagaki, Thin Solid Films 315 (1998).

${ }^{21}$ T. Zhao, H. Lu, F. Chen, S. Dai, G. Yang, and Z. Chen, J. Cryst. Growth 451, 212 (2000).

${ }^{22}$ W. Nam, H. Seo, S. C. Park, C. H. Bae, S. H. Nam, S. M. Park, and J. S. Ha, Jpn. J. Appl. Phys. 43, 11 (2004).

${ }^{23}$ C. V. Cojocaru, C. Harnagea, F. Rosei, A. Pignolet, M. A. F. van den Boogaart, and J. Brugger, Appl. Phys. Lett. 86, 183107 (2005).

${ }^{24}$ C. C. Cojocaru, C. Harnagea, A. Pignolet, and F. Rosei, IEEE Trans. Nanotechnol. 5, 5 (2006). 